\title{
Nurse-led orthopaedic clinic in total joint replacement
}

\author{
Jason CH Fan *, Carmen KM Lo, Carson KB Kwok, KY Fung
}

\section{A B S T R A C T}

Objectives: To introduce the practice of a nurseled orthopaedic clinic for managing stable patients after total hip or knee replacement and to evaluate its efficacy.

Design: Case series.

Setting: A public hospital in Hong Kong.

Patients: Patients who had stable primary total knee replacement or total hip replacement done for longer than 2 years were managed in a nurse-led total joint replacement pilot clinic.

Results: From July 2012 to March 2014, 431 patients (including 317 with total knee replacement and 114 with total hip replacement) were handled, and 408 (94.7\%) nurse assessments were independently performed. Six cases of prosthesis-related complications were diagnosed. One patient was hospitalised for prosthetic complications within 3 months after follow-up. The satisfaction rate was 100\%. From November 2012 to April 2013, an advanced practice nurse, one resident specialist, and one associate consultant independently charted Knee Society Knee Score or Harris Hip Score for the patients attending preoperative assessment clinic to check the inter-observer reliability. Overall, 23 patients with 37 knees and 11 patients with 17 hips were examined. The mean correlation coefficient between assessments by the associate consultant and advanced practice nurse was 0.912 for Knee Society Knee Score, and 0.761 for Harris Hip Score. The advanced practice nurse could achieve better or equally good correlation with associate consultant when compared with the correlation between resident specialist and associate consultant $(0.866$ and 0.521 for Knee Society Knee Score and Harris Hip Score, respectively) and with international standard.

Conclusion: Nurse-led total joint replacement clinic was safe, reliable, and well accepted by patients.

\section{Hong Kong Med J 2014;20:511-8}

DOI: $10.12809 / \mathrm{hkmj} 134150$

JCH Fan *, FHKAM (Orthopaedic Surgery)

CKM LO, MN

CKB Kwok, FHKAM (Orthopaedic Surgery)

KY Fung, FHKAM (Orthopaedic Surgery)

Department of Orthopaedics and Traumatology, Alice Ho Miu Ling Nethersole Hospital, Tai Po, Hong Kong

* Corresponding author: fchjason@netvigator.com

This study was presented at the 33rd Annual Congress of the Hong Kong Orthopaedic Association on 23 November 2013.

New knowledge added by this study

Management of stable postoperative total joint replacement patients by well-trained nurses is safe, reliable, and well accepted by patients.

Implications for clinical practice or policy

- Nurse-led clinics should be established for managing patients with total joint replacement to handle the escalating workload in Hong Kong.

- Well-structured nurse training should be organised for managing stable patients with total joint replacement in orthopaedic clinics.

\section{Introduction}

Nursing practice is moving towards both proletarianisation and professionalisation. ${ }^{1}$ The former means the transfer of some basic routine care to less-skilled assistants. The latter means advanced nursing practice with nurses handling complex health problems using advanced health assessment and intervention measures. Nurse-led clinic is one of the forms of professionalisation and has been adopted in Hong Kong since 1990s. Diabetes clinic, wound clinic, and continence clinic are the three most common nurse-led clinics in Hong Kong ${ }^{2}$ and they have demonstrated significant impact on patient outcomes. ${ }^{3}$ Orthopaedic nurses in total joint replacement (TJR) have been focusing on patient education in preoperative assessment clinics.

In order to enhance the role of orthopaedic nurses and ensure the continuity of patient care before and after TJR, a new advanced nursing practice was introduced, namely, the Ambulatory Comprehensive Arthroplasty Clinic (ACAC), a nurse-led orthopaedic postoperative clinic in TJR. 


\section{護士主導的全關節置換骨科診所 范智豪、盧嘉敏、郭嘉邦、馮貴游}

目的：引進骨科護士主導的診所來管理全髖關節或膝關節置換術後病 情穩定的患者和評估其功效。

設計：病例系列。

安排：香港一所公立醫院。

患者：完成超過兩年而穩定的初次全膝關節置換術（TKR）或髖關節 置換術（THR）患者在護士主導的關節置換試點門診管理。

結果：在2012年7月至2014年3月期間, 處理了 431 例, 當中包括 317 例TKR和 114 例THR，其中408例（94.7\%）由護士作獨立評估。有6 例為假體併發症。一名病人因假體併發症在覆診 3 個月內住院。病人 滿意度為100\%。從2012年11月到2013年4月, 一名資深護士、一名 駐院專科醫生和一名副顧問醫生分別獨立評估術前門診患者的膝關節 學會膝關節評分 (KSKS) 或Harris髖關節評分 (HHS) 來檢測觀察 者間的一致性。23名患者 ( 37 例膝蓋關節) 和 11 名患者 ( 17 例髖關 節）進行了檢查。至於在評估KSKS 和HHS方面, 副顧問醫生和資深 護士之間的平均相關係數為 0.912 和 0.761 , 而駐院專科醫生與副顧 問醫生之間於KSKS和HHS評估的相關係數分別為0.866和 0.521 。因 此, 資深護士與副顧問醫生之間於KSKS 和HHS評估的相關係數與前 者或國際標準相若甚至更佳。

結論：護士為主導的關節置換診所是安全和可靠的, 並為患者所接 受。 in ACAC for continuity of care. In part of the KSKS and HHS, physical examination is necessary. It was a difficult area for the APN and could lead to potential error and discrepancy. Therefore, part of the APN training was concentrated in this area and a separate study was launched to test her reliability.

This article aimed to introduce the practice of a nurse-led orthopaedic clinic for managing stable postoperative patients with TKR and THR and describe the outcomes in a series of cases. This also presents the result of the reliability of the APN in charting various scores.

\section{Methods}

From August 1997 to December 2013, 895 primary TKRs and 268 primary THRs were performed in Alice Ho Miu Ling Nethersole Hospital. Until 31 December 2013, 801 TKRs and 232 THRs were performed for more than 2 years and were followed up yearly in the specialist out-patient clinic. During each follow-up, the assessment included history taking and physical examination, charting KSKS or HHS, and checking radiographs. Patients who had undergone primary TKR or THR more than 2 years ago and who were assessed as being stable and minimally symptomatic by specialists were recruited in the ACAC for yearly follow-up. One day before follow-up, the AC analysed the radiographs taken in the earlier year and the finding was discussed with the APN for teaching purpose. During ACAC, the APN assessed the patients by TKR (Fig 1) or THR (Fig 2) questionnaire, charted KSKS or HHS, interpreted follow-up radiographs, and then educated the patients on care of and precautions with the prosthesis. The findings were recorded in consultation notes in the computer medical system (CMS). Patients were managed according to the workflow (Fig 3). When the TJR specialist was consulted on-site for any problem related to the prosthesis, minor procedures like knee aspiration could be done by the specialist at the same consultation. After the clinic, the questionnaires and the radiographs were screened by the specialist for any significant problems.

The questionnaires were collected and the consultation notes in CMS were studied. The relevant data were then summarised and described in an Excel file, including the number of cases of TKRs and THRs, the number of on-site specialist consultations, the number of prosthesis-related complications diagnosed by the APN, the number of patients referred to orthopaedic clinic and other health care professionals, the number of medication prescriptions, patients' satisfaction, and patients' acceptance of consultation without medication. Patients' waiting time for the clinic was also obtained by calculating the difference between the consultation time and the allocated time slot. The CMS was also checked for any hospital admission 
Name:

OT date:

Left/right total knee replacement

For recent 1 year

(1) How would you describe the pain you usually have in your knee?

1 None (go to Q6) 2 Very mild 3 Mild 4 Moderate 5 Severe

(2) What is/are the site(s) of pain?
1 Popliteal fossa 2 Lateral
3 Medial
4 Anterior
5 Diffuse

(3) Mark the site of tenderness on palpation

1 None 2 Medial femoral condyle 3 Lateral femoral condyle 4 Medial tibial edge

5 Lateral tibial edge 6 Medial cruciate ligament 7 Lateral cruciate ligament 8 Patella tendon

(4) What is/are the nature(s) of pain?

1 Radiation pattern from back to calf 2 Mechanical 3 Triphasic pattern 4 Rest pain 5 Night pain

(5) How often have you been awakened by pain from your hip in bed at night?

1 None 2 Less than once per week 3 Once per week 4 Many nights 5 Every night

(6) Is there any problem with the surgical wound?

1 None 2 Mild tender scar 3 Redness around scar 4 Discharge/sinus

(7) Is there any swelling of the knee?

1 None 2 Localised 3 Recurrent diffuse 4 Persistent diffuse

(8) Are you losing the range of knee movement?

1 None 2 Occasional 3 Gradual 4 Sudden

(9) Is there any noticeable deformity of limb?

1 None 2 Very mild 3 Mild 4 Moderate 5 Severe

(10) Have you noticed any painful jumping of patella with active knee extension? 1 None 2 Rarely 3 Sometimes 4 Often 5 Frequently

(11) Have you had any giving-way and unstable feeling of the knee joint? 1 None 2 Rarely 3 Sometimes 4 Often 5 Frequently

(12) Is there any increased limping? 1 None 2 Mild 3 Occasional 4 Persistent 5 Significant

(13) How would you describe the nature of your job? 1 Unemployed/retired 2 Sedentary 3 Light duty 4 Heavy duty 5 Very heavy duty

(14) How would you rate the total knee replacement when compared with the preoperative status? 1 Extremely satisfied 2 Satisfied 3 Fair 4 Not satisfied 5 Extremely unsatisfied

FIG I. Questionnaire for total knee replacement follow-up

or clinic attendance for any problems related to TJR after ACAC follow-up.

From November 2012 to April 2013, an APN, one resident with specialist qualification (RS), and one AC independently charted KSKS or HHS for patients attending the preoperative assessment clinic. Knee Society Knee Score is composed of function score (FS) and knee score (KS)_FS is made up of three components and KS is made up of seven components. Harris Hip Score is composed of 17 components. Overall, 23 patients with 37 knees and 11 patients with 17 hips were examined. In order to analyse the inter-rater reliability between the AC and APN (comparison A), between AC and RS (comparison B), and between RS and APN (comparison $\mathrm{C}$ ), each component of the KSKS and HHS was analysed for single-measure intraclass correlation coefficient (ICC) and statistical significance using the Statistical Package for the Social Sciences (Windows version 15.0; SPSS Inc, Chicago [IL], US). To see any statistically significant difference between ICCs among the three groups and between comparison $\mathrm{A}$ and international standards, ${ }^{4,5}$ Fisher's z-transformation was performed by online calculator (www.vassarstats. net/rdiff.html). 
Name:

OT date:

Left/right total hip replacement

Bearing surface: metal on polyethylene / ceramic on polyethylene / metal on metal / ceramic on ceramic

For recent 1 year

(1) How would you describe the pain you usually have from your hip?

1 None (go to Q5) 2 Very mild 3 Mild 4 Moderate 5 Severe

(2) What is/are the site(s) of pain?

1 Back/buttock 2 Posterior thigh 3 Greater trochanter 4 Groin 5 Anterior thigh

(3) What is/are the nature(s) of pain?

1 Radiation pattern from back to calf $\quad 2$ Mechanical $\quad 3$ Triphasic pattern $\quad 4$ Rest pain $\quad 5$ Night pain

(4) How often have you been awakened by pain from your hip in bed at night?

1 None 2 Less than once per week 3 Once per week 4 Many nights 5 Every night

(5) Is there any problem with the surgical wound?

1 None 2 Mild tender/itchy scar 3 Redness around scar 4 Swollen wound 5 Discharge/sinus

(6) Is there any increased limping?

1 None 2 Mild 3 Occasional 4 Persistent 5 Significant

(7) Is there any noticeable shortening of limb length?

1 None 2 Very mild 3 Mild 4 Moderate 5 Severe

(8) Have you noticed any noise from your total hip replacement?

1 None 2 Rarely 3 Sometimes 4 Often 5 Frequently

(9) Have you had any feeling of the hip joint going out of socket?

1 None 2 Rarely 3 Sometimes 4 Often 5 Frequently

(10) How often have you done deep squatting/leg crossing?

1 None 2 Rarely 3 Sometimes 4 Often 5 Frequently

(11) What type of transport have you most often used?

1 Private car 2 MTR 3 Taxi 4 Mini bus 5 Bus

(12) How would you describe the nature of your job?

1 Unemployed/retired 2 Sedentary 3 Light duty 4 Heavy duty 5 Very heavy duty

(13) How would you rate the total hip replacement when compared with the preoperative status?

1 Extremely satisfied 2 Satisfied 3 Fair 4 Not satisfied 5 Extremely unsatisfied

FIG 2. Questionnaire for total hip replacement follow-up

\section{Results}

In the initial period from January to June 2012, on-site specialist consultation was necessary in 21 (22.8\%) cases out of the 92 cases (68 TKRs and 24 THRs). From July 2012 to March 2014, a total of 431 patients (including 317 TKRs and 114 THRs) were managed and 408 (94.7\%) nurse assessments were independently performed. Six cases of prosthesisrelated complication were diagnosed including two cases of patellar clunk in TKR, two cases of TKR loosening, and two cases of THR loosening. Number of referrals to other orthopaedic clinics and health care professionals is shown in the Table. Among the 523 patients on ACAC follow-up, 131 (25.0\%) requested medications. Average patient waiting time improved over the study period (26 minutes in December 2012, 18 minutes in April 2013, and 14 minutes in March 2014). Of these 523 patients, 485 patients were interviewed; 354 were extremely satisfied and 131 were satisfied with ACAC followup by the nurse, and 373 (76.9\%) patients accepted follow-up without drug prescription.

One patient who had undergone right TKR 12 years ago was hospitalised at 3 months after nurse clinic follow-up because of sudden onset of right knee effusion. X-ray showed no sign of prosthesis 


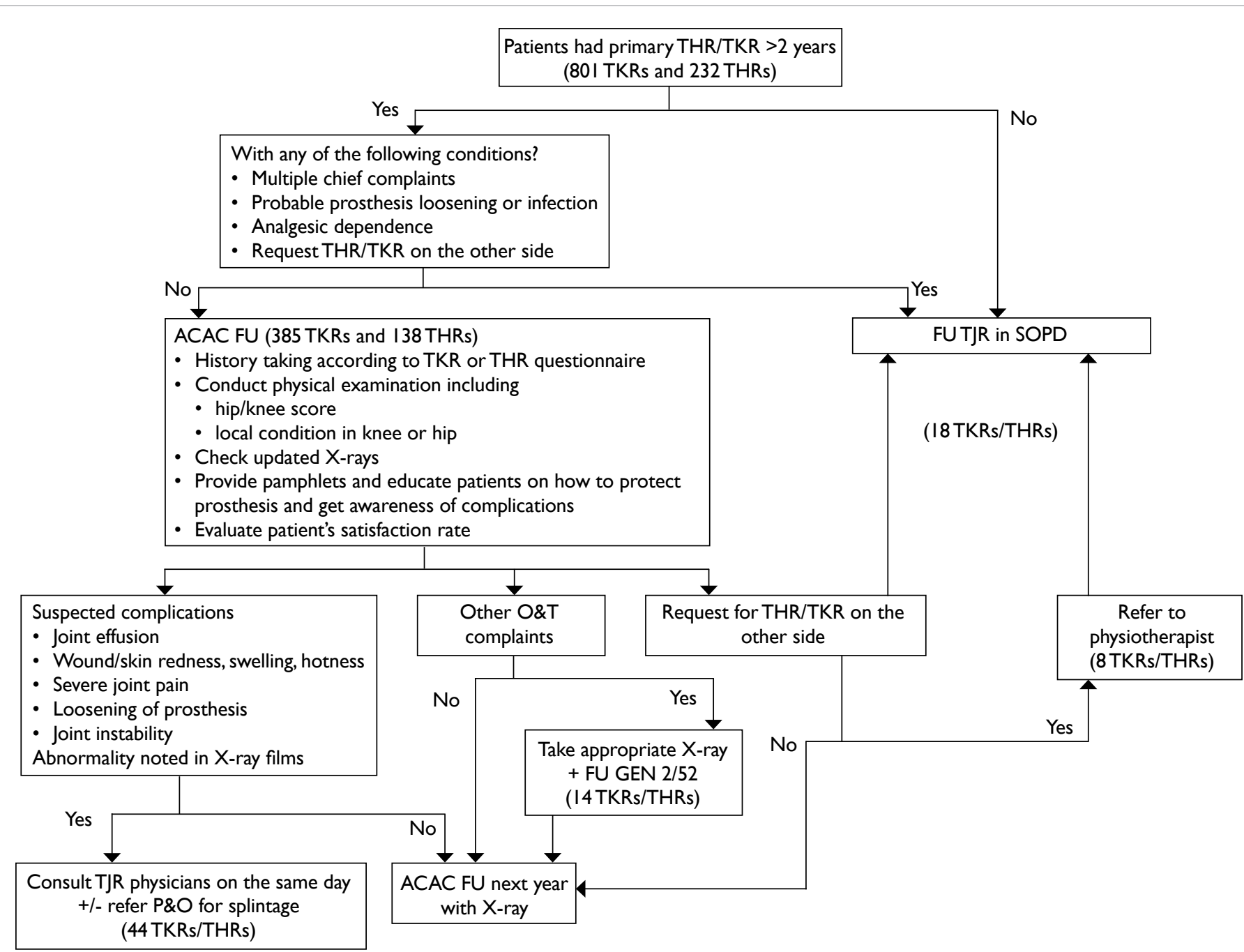

FIG 3. Workflow of patient arrangement

Abbreviations: ACAC = Ambulatory Comprehensive Arthroplasty Clinic; FU = follow-up; GEN = general; O\&T = orthopaedics and traumatology;

$\mathrm{P} \& \mathrm{O}=$ prosthetics and orthotics; $\mathrm{SOPD}=$ specialist out-patient department; $\mathrm{THR}=$ total hip replacement; $\mathrm{TJR}=$ total joint replacement; TKR = total knee replacement

TABLE. Number of patients referred to other clinics and treatment centres

\begin{tabular}{lc}
\hline Destination of referral & No. of patients \\
\hline Total Joint Replacement Clinic & 18 \\
Other O\&T clinics & 14 \\
Physiotherapy & 8 \\
Occupational Therapy & 2 \\
Prosthetics and Orthotics & 1 \\
\hline
\end{tabular}

Abbreviation: O\&T = Orthopaedics and Traumatology

loosening. Surgical exploration of the right knee showed catastrophic wear of the polyethylene insert while the prosthesis was stable. Two patients at 6 months after nurse assessment attended general outpatient clinic for getting analgesics.

\section{Study of reliability of the advanced practice nurse in charting Knee Society Knee Score and Harris Hip Score}

The result for KSKS is shown in Figure 4. The mean ICCs were 0.912 (range, 0.660-0.987), 0.866 (range, 0.735-0.974), and 0.851 (range, 0.599-0.996) for comparisons A, B and C, respectively. The lowest ICC among all the components in KSKS was that of mediolateral stability (0.599-0.797). When comparing with those of Bach et al, ${ }^{4}$ the ICCs of FS and all its components for comparison A were significantly better. This was also the case for ICCs of three of the five computable components of KS and that of KS.

The result for HHS is shown in Figure 5. The mean ICCs were 0.761 (range, 0.211-1) for comparison A, 0.521 (range, 0.101-0.940) for 


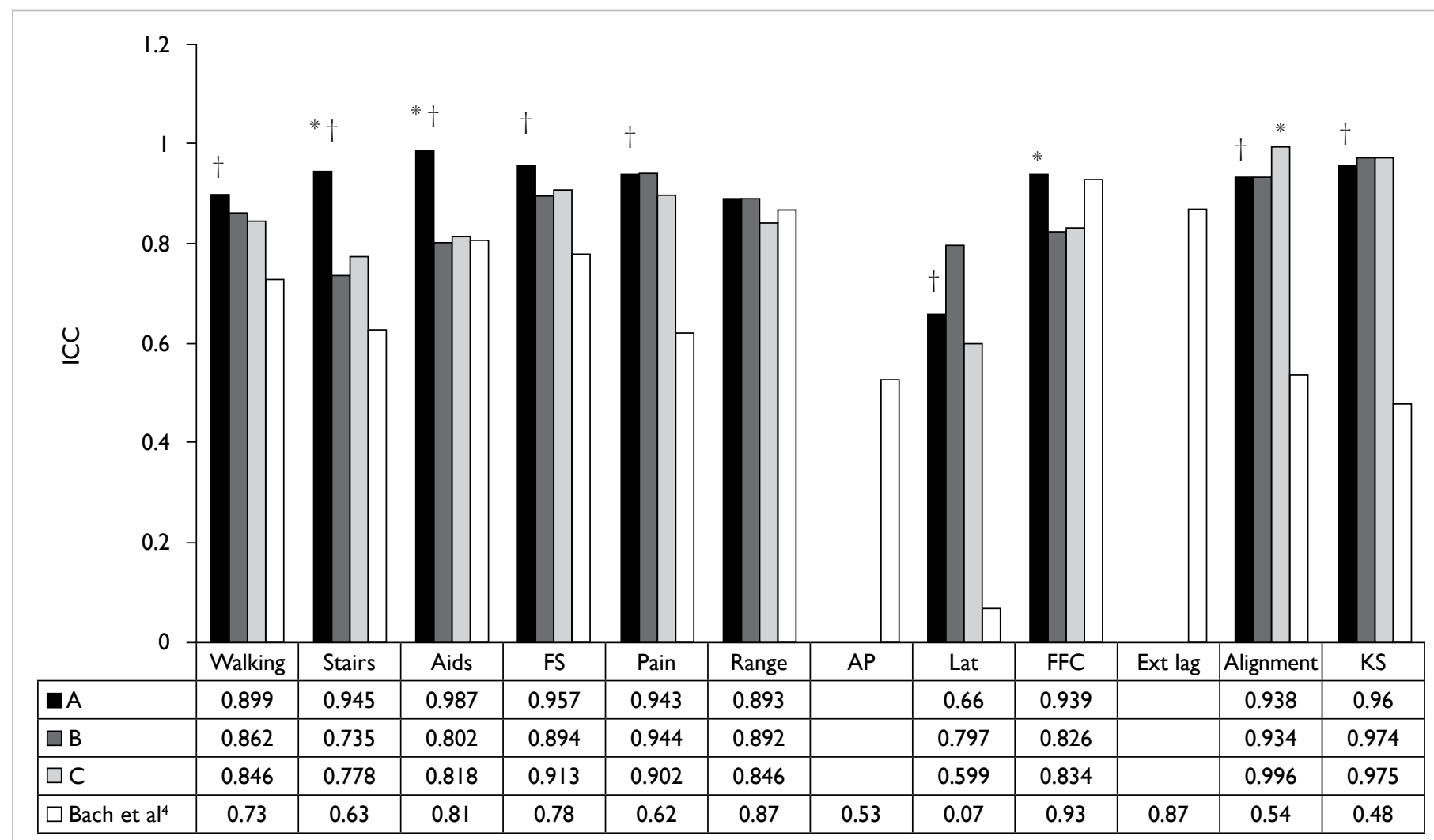

FIG 4. Results for Knee Society Knee Score

The ICCs for comparison among A, B, and C in AP and Ext lag could not be computed because one of the variables was constant. All the available ICCs

for comparison between $A$ and $C$ were statistically significant $(P<0.05)$

* When comparisons A, B, and C were compared with each other, the ICC of the labelled group was statistically the best (P<0.05)

+ Comparison A was statistically better than Bach et $\mathrm{l}^{4}(\mathrm{P}<0.05)$

Abbreviations: $A P=$ maximum anteroposterior translation; Ext $=$ extension; FFC $=$ fixed flexion contracture; FS $=$ function score; ICC $=$ intraclass

correlation coefficient; $\mathrm{KS}=$ knee score; Lat = maximum mediolateral angular laxity

comparison $\mathrm{B}$, and 0.481 (range, -0.231 to 0.940 ) for comparison $\mathrm{C}$. The ICCs for charting total HHS were 0.964, 0.747, and 0.722 for comparisons A, B and C, respectively. When the ICC of comparison A (0.964) was compared with that in the study by Kirmit et $\mathrm{al}^{5}$ (0.91), the difference was found to be statistically insignificant $(\mathrm{P}=0.124)$.

\section{Discussion}

In the current study, the well-trained APN could independently handle $94.7 \%$ of the stable postoperative cases in TJR after the initial learning phase. She could successfully diagnose six cases of prosthesis-related complications out of all the 523 patients she handled. One patient who was asymptomatic at the ACAC follow-up presented with sudden onset of right knee effusion, and showed catastrophic wear of polyethylene. Nurse-led clinic in TJR was, therefore, safe. It was well accepted by patients with a $100 \%$ satisfaction rate. As the APN grew more confident and gained more experience, she also became more efficient. This, together with improvement in workflow in taking radiographs, led to progressive shortening of patient waiting time from 26 minutes in the initial phase (December 2012) to 14 minutes in March 2014. It could greatly relieve the burden of the specialist clinic. Within 2 years, 523 patient attendances were handled by the APN. About five to seven cases were seen in each session of this clinic. Moreover, more detailed patient education, which is not possible in the busy specialist clinic, can be provided to patients who may have forgotten the details given several years ago before the operation.

Bach et $\mathrm{al}^{4}$ studied the inter-observer correlation of four commonly used TKR outcome scoresHungerford score, Hospital for Special Surgery score, Knee Society score, and Bristol score. Two experienced orthopaedic surgeons independently assessed 118 TKRs in 92 patients. The correlation coefficient for mediolateral knee stability was the lowest among all the components of all scoring systems (0-0.38). This was due to the difficulty in physical examination and proper measurement with goniometer at the same time. For all the comparisons in this study, we encountered the same difficulty 


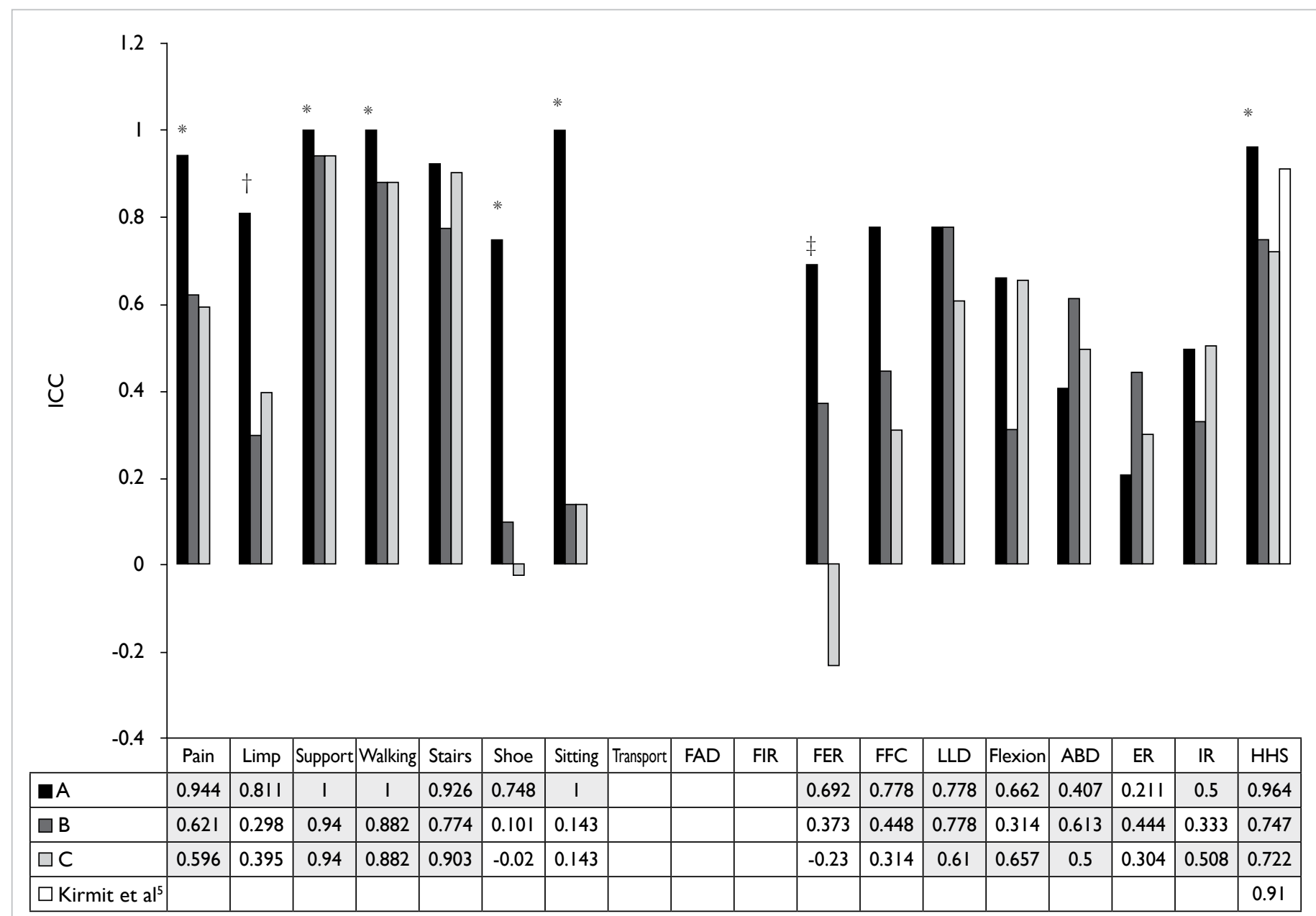

FIG 5. Results for Harris Hip Score

The intraclass correlation coefficients (ICCs) for comparison among A, B, and C in Transport, FAD, and FIR could not be computed because one of the variables was constant. The ICCs in grey boxes were statistically significant $(P<0.05)$

* When comparisons A, B, and C were compared with each other, the ICC of the labelled group was statistically the best (P<0.05)

† Comparison A was only statistically better than comparison $B(P<0.05)$

‡ Comparison A was only statistically better than comparison $\mathrm{C}(\mathrm{P}<0.05)$

Abbreviations: $\mathrm{ABD}=$ abduction range; $\mathrm{ER}=$ external rotation range; $\mathrm{FAD}=$ fixed adduction contracture; FER $=$ fixed external rotation contracture; FFC = fixed flexion contracture; FIR = fixed internal rotation contracture; HHS = Harris Hip Score; IR = internal rotation range; LLD = limb length discrepancy

and found the same finding of lowest correlation coefficient for mediolateral stability $(0.599-0.797)$ among all the components in KSKS. Kirmit et $\mathrm{al}^{5}$ evaluated the inter-observer reliability of five different hip scores including HHS. Three physiotherapists assessed 48 hips with osteoarthritis in 35 patients. The correlation coefficients ranged from 0.82 to 0.91 for HHS, which was comparable to the result in our study (0.722-0.964). With proper training of the APN by the AC in this hospital, she could achieve better or equally good correlation with the $\mathrm{AC}$ as compared with an orthopaedic specialist and international standard for charting KSKS and HHS.

In order to match the evolution of the health care environments and patient care needs, the roles and responsibilities of APNs have been reshaping. ${ }^{6}$ They have active and important places in taking care of patients from various specialties. Their contribution by running nurse-led clinics has been shown to be tremendous in and outside Hong Kong. They add value to patient care and complement specialist clinics., ${ }^{3,-9}$ Over $80 \%$ of their work are independent of or interdependent with physicians and involve skills such as adjusting medications, and initiating therapies and diagnostic tests according to protocols. ${ }^{3,10}$ To further advance professionalisation of nursing practice, Newey et $\mathrm{al}^{11}$ reported the training of nurse practitioners to provide initial assessment in clinics, perform carpal tunnel release, and manage these patients in postoperative follow-up.

Shiu et $\mathrm{al}^{12}$ pointed out four boundaries and six hindering factors for expanding advanced nursing practice in nurse-led clinics. The former included community-hospital, wellness- 
illness, public-private, and professional-practice boundaries. The latter included stakeholder and public awareness of advanced nursing practice role in nurse-led clinics, provision of advanced specialty education programmes, organisational support, multidisciplinary collaboration, and changing health care context and provision. When ACAC was commenced, professional-practice boundary was the first and the most important hurdle. The APN was directly coached by an AC about various aspects in TJR. The TKR and THR questionnaires and workflow protocol were devised to facilitate the patient care process. She was authorised to order standard radiographs according to the region of interest, and make referral to physiotherapy, occupational therapy, prosthetics and orthotics, and the general orthopaedic clinic. However, nurse prescription was not possible and doctor prescription was necessary in $25 \%$ of the cases. Getting help from doctors was also required in a few cases for writing referral letters to medical departments, applying for car park permits for the disabled, applying for public housing, and signing disability allowance forms. In order to solve these remaining problems, there should be appropriate legislation to redefine the professional code of nursing practice, and organisational support to offer a clear policy for nurse prescribing. ${ }^{12}$ Stakeholder and public awareness should be aroused to allow inter-departmental referral and granting public certification.

The second hurdle was the provision of advanced specialty education programmes. Currently, there is no programme or course in universities and Hospital Authority teaching nurses about TJR. Direct coaching was chosen as the training method, and continuous education was provided to broaden her exposure. This may be less than ideal and a complete curriculum was not present. If the nurse-led clinic were to be promoted and accepted as the method to deal with the escalating workload from various joint replacement centres, the Coordinating Committee (COC), which is one of the Hospital Authority Head Office committees for clinical service, in orthopaedics and traumatology has to collaborate with $\mathrm{COC}$ in nursing to formulate a good training programme for nurses with special interest in TJR. Knowledge of pharmacology is also necessary for nurse prescription. The nursing schools in various universities should revise the curriculum to make nurse-led orthopaedic clinics feasible and safe.

\section{Conclusion}

The success of nurse-led postoperative clinic in TJR is multifactorial including the experience and dedication of the APN, support of the trainer specialist and department, a good working guideline and protocol, and support of other health care professionals. Its running is not perfect yet because the specialty nurse cannot prescribe medications and she is not community-recognised to sign legal documents. However, such a clinic should be established in Hong Kong to align with the development of joint replacement centres in Hospital Authority. Apart from the preoperative assessment clinic and postoperative follow-up clinic, the trained specialist nurse can also play important roles in other stages of patient care. This requires further exploration and collaboration with other health care professionals.

\section{Acknowledgements}

We thank Ms Amy MY Cheng and Winnie YC Lam for valuable inputs when setting up the nurse-led clinic.

\section{References}

1. Wong FK. Health care reform and the transformation of nursing in Hong Kong. J Adv Nurs 1998;28:473-82.

2. Hospital Authority Annual Plan 2008-2009. Available from: http://www.ha.org.hk/visitor/ha_visitor_index.asp?Parent_ ID $=100 \&$ Content_ID=300\&Dimension=100\&Lang=ENG. Accessed Aug 2010.

3. Wong FK, Chung LC. Establishing a definition for a nurseled clinic: structure, process, and outcome. J Adv Nurs 2006;53:358-69.

4. Bach CM, Nogler M, Steingruber IE, et al. Scoring systems in total knee arthroplasty. Clin Orthop Relat Res 2002;399:184-96.

5. Kirmit L, Karatosun V, Unver B, Bakirhan S, Sen A, Gocen $Z$. The reliability of hip scoring systems for total hip arthroplasty candidates: assessment by physical therapists. Clin Rehabil 2005;19:659-61.

6. Bonsall K, Cheater FM. What is the impact of advanced primary care nursing roles on patients, nurses and their colleagues? A literature review. Int J Nurs Stud 2008;45:1090-102.

7. Larsson I, Bergman S, Fridlund B, Arvidsson B. Patients' experiences of nurse-led rheumatology clinic in Sweden: a qualitative study. Nurs Health Sci 2012;14:501-7.

8. Slight C, Marsden J, Raynel S. The impact of a glaucoma nurse specialist role on glaucoma waiting lists. Nurs Prax N Z 2009;25:38-47.

9. Kirkwood BJ, Pesudovs K, Latimer P, Coster DJ. The efficacy of a nurse-led preoperative cataract assessment and postoperative care clinic. Med J Aust 2006;184:278-81.

10. Ng SL, Tse YB, Ho KL, Yiu MK. Efficacy of a Urology Nurse-led Clinic in Queen Mary Hospital of Hong Kong. Proceedings of the 11th Asian Congress of Urology of the Urological Association of Asia; 2012 Aug 22-26; Pattaya, Thailand. Int J Urol 2012;19(Suppl 1):432 abstract no. OP2512-07.

11. Newey M, Clarke M, Green T, Kershaw C, Pathak P. Nurseled management of carpal tunnel syndrome: an audit of outcomes and impact on waiting times. Ann R Coll Surg Engl 2006;88:399-401.

12. Shiu AT, Lee DT, Chau JP. Exploring the scope of expanding advanced nursing practice in nurse-led clinics: a multiplecase study. J Adv Nurs 2012;68:1780-92. 